\title{
MicroRNA-134/MicroRNA-200a DERIVED SALIVARY EXOSOMES ARE NOVEL DIAGNOSTIC BIOMARKERS OF ORAL SQUAMOUS CELL CARCINOMA
}

\author{
Amina Fouad Farag ${ }^{*}$, Dina Sabry**, Nadia Fathy Hassabou* \\ and Yasmine Alaa El-Din*
}

\begin{abstract}
Background and Aim: Oral squamous cell carcinoma is by far one of the most common oral cancers. The success of early detection of cancer using recent modalities such as collected human saliva can guarantee a high success rate of treatment. This study aimed to investigate the expression of microRNAs and interleukins in exosomes as potential salivary biomarkers and to evaluate their accuracy in early detection of oral cancer.
\end{abstract}

Material and methods: Samples of saliva were collected from 37 patients divided into 6 healthy control, 17 smokers and 14 patients with different grades of oral squamous carcinoma. Exosomes were isolated from $3 \mathrm{ml}$ saliva using ultracentrifugation protocol and were prepared for electron microscopy characterization. Expression of microRNA-200a and microRNA-134 was analyzed by qRT-PCR. IL-1 $\beta$ and IL-8 concentrations were also assessed in isolated salivary exosomes. All data array of salivary isolated tested biomarkers was represented as mean values and standard deviation.

Results: Concentrations of both salivary IL-1 $\beta$ \& IL-8 demonstrated a highly significant increase in cancer patients compared to smoker and control groups $(\mathrm{p} \leq 0.00001)$. Additionally, microRNA-200a and microRNA-134 were upregulated and revealed a highly significant increase in cancer patients in comparison to other groups $(\mathrm{p} \leq 0.00001)$. No significant changes were observed between different grades of oral squamous cell carcinoma.

Conclusion: Isolated salivary exosomes provide a stable and non-invasive route for evaluation of different salivary biomarkers which can be a useful tool in early detection of oral cancer.

KEYWORDS: Salivary biomarkers, exosomes, microRNA, interleukins, oral squamous cell carcinoma.

* Lecturer of Oral and Maxillofacial Pathology, Faculty of Dentistry, October 6 University, Egypt.

** Professor of Medical Biochemistry, Faculty of Medicine, Cairo University, Egypt. 


\section{INTRODUCTION}

Identification of cancer biomarkers in liquid biopsies collected from body biofluids as saliva, blood, urine and cerebral spinal fluid offers a promising reliable alternative method to invasive tissue biopsy. Among all these biofluids, saliva as a diagnostic fluid offers advantages over other biofluids due to being a cheap method, non-invasive, accessible and easily-collectable media which facilitates the early detection of cancer, expecting prognosis, observing status of the patient's after therapy in oral cancer $^{(1,2)}$.

Lately, the interest in the biology of extracellular vesicles known as exosomes (EXOs) has increased greatly in which such salivary analytes are released and carried. EXOs are regarded as a novel mechanism by which cancer cells and virally infected cells can regulate their micro-environment ${ }^{(3)}$.

The salivary EXOs are membrane-bonded microvesicles of endocytic origin whose diameter range from $40-100 \mathrm{~nm}$ and are secreted into the extracellular space by exocytosis. They were initially thought to be a way for cells to get rid of unnecessary proteins, but are nowadays considered as mediators of intercellular signaling and cellular homeostasis through RNA and functional protein exchange ${ }^{(4,5)}$. Thus, it is strongly suggested those salivary EXOs as a potential source for cancer biomarkers and raises the demand to find applicable ways for their simple isolation and clinical analysis ${ }^{(6)}$.

Several mRNA transcripts are up-regulated in the saliva of oral squamous cell carcinoma (OSCC) patients among which IL- 8 and IL- $1 \beta$ that play an important role in cell cycle arrest, replication, angiogenesis and cell adhesion ${ }^{(7)}$.

IL-8 is an important pro-inflammatory chemokine which expresses and is released by the neutrophils and macrophages. In addition, they are triggered by various stimuli which activate the nuclear factor-Kappa-B pathway while IL-1 $\beta$ is a well-known potent promoter of carcinogenesis. Many evidences reported that cancer progression correlates with an increase in IL- 8 and IL- $1 \beta$ release, suggesting that they are potential biomarkers assist patients' cancer risk and predict cancer prognosis ${ }^{(8-10)}$.

MicroRNAs (miRNAs) one of the most identified genetic materials in EXOs. They are short (1925 nucleotides) non-coding RNAs molecules that act as regulatory gatekeepers of coding genes ${ }^{(11)}$.

Interestingly, their encapsulation in EXOs makes them more stable upon release and protects them from enzymatic degradation ${ }^{(12,13)}$. Moreover, those salivary miRNAs analytes harbored and released in EXOs can precisely discriminate poorly differentiated oral tumors better than mRNA which revealed highly inaccurate results on the same samples making them more useful for characterizing solid tumors and in turn more promising and very powerful salivary cancer biomarkers ${ }^{(12)}$.

MicroRNA-134 (miR-134) is originally a brainspecific miRNA located in a miRNA cluster consisting of 44 miRNAs on chromosome 14q32.9. Multiple pathophysiological processes of many tumors are affected by expression of miR-134 which acts as tumor suppressor gene repressing various targets to inhibit the tumor oncogenicity ${ }^{(14,15)}$.

In OSCC, miR-134 targets Programmed Cell Death 7 (PDCD7) which is one of programmed cell death proteins involved in apoptotic accelerating activity ${ }^{(16)}$ which in turn trans-activates the E-cadherin expression which is considered as progression marker to drive oncogenicity in OSCC ${ }^{(14)}$.

MicroRNA-200a (miR-200a) is one of members of miR-200 family which is a group of tumor suppressor miRNAs involved in the epithelialmesenchymal transition (EMT) regulation, selfrenewal repression, cancer stemlike cells differentiation and chemoresistance reversal ${ }^{(17)}$.

Downregulation or even inhibition of miR-200a within cancer cells allows ZEB1/ZEB2 expression to be maintained by a positive regulatory loop which reduces expression of E-cadherin, increases 
vimentin expression, induces the EMT mechanism and in turn enables tumor invasion, metastasis and resistance to therapy ${ }^{(18)}$.

There are limited literatures reporting the analysis of biomarkers in salivary EXOs of patients suffering from OSCC and their precise role in carcinogenesis and the development of oral cancer from oral potential malignant disorders (OPMDs) has been relatively unexplored.

With this backdrop, this study was designed to evaluate the salivary IL- 8 , IL- $1 \beta$, miR-200a and miR-134 isolated from salivary EXOs in patients with different grades of OSCC, heavy smokers as high risk group patients suggestive for transformation to OPMDs and healthy controls with the ultimate aim was to evaluate their efficacy as potential biomarkers for oral cancer development. To the best of our knowledge, this was the first study of this type to be carried out in the Egyptian population.

\section{MATERIAL AND METHODS}

\section{Patient selection}

The current study consisted of 37 patients divided into 6 normal healthy controls, 17 smokers and 14 patients previously histopathologically diagnosed to suffer from OSCC presented to our outpatient clinics of October 6 university. The OSCC consisted of 9 well-differentiated and 5 poorly differentiated cases. The participant population consisted of 11 females and 26 males. Mean age was ranging from 30- 60 years old. Our exclusion criteria included existence of any systemic, autoimmune disease, consumption of drugs may affect salivary flow and recent surgical operations.

All the participant subjects signed the institutional review board-approved consent form agreeing to serve as saliva donors for the experiment. This study was approved by Research Ethics Committee at Faculty of Dentistry, October 6 University, Giza, Egypt (No. RECO6U/3-2019) and conducted following the principles of the Declaration of Helsinki.

\section{Saliva collection}

As previously reported by Gai et al., ${ }^{(12)}$ all volunteers were asked to refrain from eating and drinking 3 hours before collection to avoid any salivary stimulation before the test. Unstimulated saliva was collected from all tested groups and control group in the morning which is the best time for sample collection. All saliva samples $(5 \mathrm{ml} / \mathrm{each})$ were collected by passive drooling and the participants were asked to rinse their oral cavity with water before collection. The specimens were collected into as sterilized containers. All samples were frozen at $-20^{\circ} \mathrm{C}$ until for the processing.

\section{EXOs isolation}

One of the fundamental cellular process occurring in living cell is salivary EXOs release whose diameter is less than $<100 \mathrm{~nm}$. The EXOs in current were isolated using ultracentrifugation protocol from $3 \mathrm{ml}$ saliva. Saliva samples obtained from patients with OSCC, smoker and healthy controls were centrifuged at room temperature for $15 \mathrm{~min}$ at $3000 \mathrm{~g}$ in order to remove bacterial cells and debris. EXOs were precipitated by the recentrifugation of the cell-free supernatant for $1 \mathrm{~h}$ at $4^{\circ} \mathrm{C}$ at $100,000 \times$ g (Beckman Coulter Optima L $90 \mathrm{~K}$ ultracentrifuge $)^{(19)}$. Bradford method (BioRad, Hercules, CA) was used to quantify protein content. The resuspension of pellet for transmission electron microscopy (TEM) analysis and characterization was performed in $600 \mu \mathrm{L}$ of Lysis/Binding Buffer or $100 \mu \mathrm{L}$ of glutaraldehyde (Zymoresearch QuickgRNA $^{\mathrm{TM}}$ MiniPrep kit, USA) and stored at $-80^{\circ} \mathrm{C}$ for subsequent RNA extraction.

\section{EXOs characterization}

Preparation of EXOs for electron microscopy characterization were performed by diluting them in $145 \mu 1$ phosphate buffer saline (PBS) containing $0.2 \%$ paraformaldehyde (w/v) where $10 \mu 1$ was administered for $7 \mathrm{~min}$ to a formvar-carbon-coated 300 mesh grid (Electron Microscopy Sciences, Hatfield, USA) before $1.75 \%$ uranyl acetate staining $(\mathrm{w} / \mathrm{v})$. 
Samples then were left to dry for $2 \mathrm{~h}$ at room temperature and imaged by TEM at $100 \mathrm{kV}$ afterwards (CM-10, Philips, Eindhoven, The Netherlands) ${ }^{(20)}$.

\section{RNA extraction}

Trizol Isolation Kit was used to extract miRNAs from purified EXOs (Zymoresearch Quick-gRNA ${ }^{\mathrm{TM}}$ MiniPrep kit, USA) according to the manufacturer's instruction. Nanodrop ND-1000 (Thermo Fisher Scientific) used for measuring RNA concentration where no contaminations were observed at ratio 260/230 and 260/280.

\section{Analysis of miRNA expression by qRT-PCR}

TaqMan ${ }^{\circledR}$ MicroRNA Reverse Transcription Kit (Applied Biosystem) was used to reversely transcribe total RNA (50 ng) to cDNA to be pre-amplified using a Biometra Thermal Cycler (Thermo Fisher Scientific) with Megaplex ${ }^{\mathrm{TM}}$ RT Primers, (Thermo Fisher Scientific). Assessment of miRNAs (miR200a and miR-134) expression of in all studied groups was done. To exclude contamination and ensure quantitation results, a negative control was used, and all samples were run in duplicate. $2^{-\Delta \Delta c t}$ method used to normalize the results of miRNA by using RNU6B as endogenous control.

A $20 \mu \mathrm{l}$ final reaction mix of qRT-PCR composed of $10 \mathrm{ng}$ of cDNA, $20 \mathrm{nM}$ miRNA-specific primer, nuclease free water (Qiagen), $10 \mu \mathrm{l}$ QuantiTect SYBR Green PCR Master Mix (Qiagen) and $50 \mathrm{nM}$ miScript Universal Primer (Qiagen) was prepared then the analysis was performed using the RealTime Thermal Cycler StepOne plus (Applied biosystem, Foster city volume, USA).

\section{ELISA}

IL-1 $\beta$ (PicoKine ${ }^{\mathrm{TM}}$ ELISA Catalog number: MBS175901, MyBiosource.com) and IL-8 (Cat. No: MBS013419, MyBiosource.com) concentrations were assessed in isolated salivary EXOs. Procedure was followed according to manual instructions.

\section{Statistical analysis}

All data array of salivary isolated tested biomarkers was represented as mean values and standard deviation using SPSS (Statistical Package for Social Sciences) 10.3 software. One-way analysis of variance test (ANOVA) and Student's t-tests were used to compare the mean values between the studied groups. P-value was considered highly significant when $\leq 0.01$ and significant when $\leq 0.05$. Pearson correlation test was also used. $\mathrm{R}$ value was considered weak relation when the value was near to zero.

\section{RESULTS}

\section{Nanoscale Structure of Salivary EXOs on TEM analysis}

On TEM analysis, extracellular vesicles mainly EXOs isolated from all participants' saliva samples through charge-based precipitation confirmed the EXOs characteristic shape, dimensions and aspects.

The salivary EXOs derived from OSCC and smoker patients appeared as a heterogeneous population of either bulging/circular or irregular vesicles showed variable distribution of vesicle size (30 to $400 \mathrm{~nm}$ ). The smaller EXOs showed denser core region compared to large ones. A more irregular morphology of EXOs with varying shapes and vesicle large aggregation were observed in OSCC samples compared to smoker ones.

Aggregates of several vesicles clumping in addition to numerous hollow large EXOs lacking the typical dense core region were seen in control group (Fig. 1a and b). However, EXOs derived from normal healthy controls appeared as discrete circular homogeneous vesicular bulging structures with a size ranging from $(60-90 \mathrm{~nm})$.

The less-dense vesicle periphery with the moredense core region gave rise to a distinct phase contrast without any apparent intervesicular fusion or aggregation (Fig. 1c). 


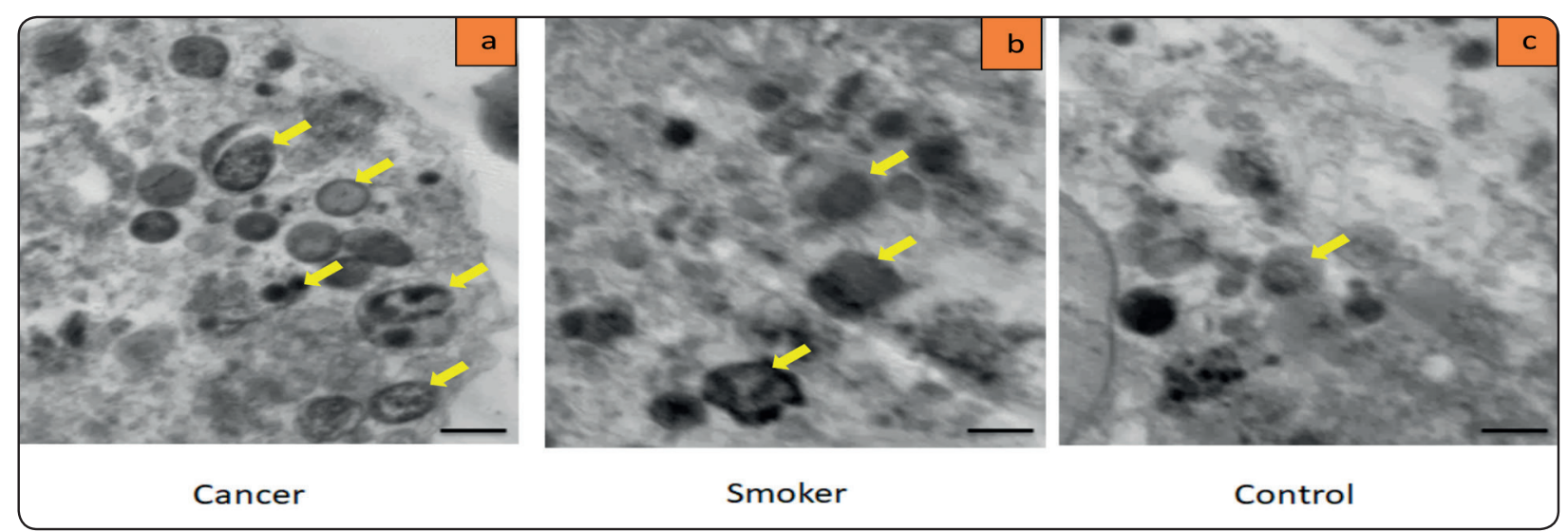

Fig. (1) Characterization of isolated salivary EXOs by TEM analysis (a) Heterogeneous higher concentration and bigger size of EXOs with irregular morphology, varying shapes, vesicle aggregates and prominent nucleosomes in OSCC samples. (b) Samples of smoker patients showing slight reduction in the size and concentration of isolated EXOs with less irregular morphology and less prominent nucleosomes compared with OSCC ones. (C) while the isolated EXOs from saliva of healthy control group demonstrated the minimum amount and the smallest size of salivary EXOs in a comparison to the previously mentioned groups.

Thus, the size and concentration of EXOs were slightly increased in OSCC patients compared to smoker patients while highly increased in a comparison to healthy control group.

\section{IL-1 $\beta$ and IL-8 expression by ELISA}

Concentrations of salivary IL-1 $\beta$ in isolated EXOs demonstrated a highly significant increase in OSCC patients than smoker and control groups $(\mathrm{p} \leq 0.00001)$. Also, it was found that salivary IL-8 was significantly higher in OSCC patients compared to smoker patients and control group $(\mathrm{p} \leq 0.00001)$, (table 1, graph 1). Significant difference was observed between analyzed salivary IL- $1 \beta$ and IL- 8 concentrations in all tested groups $(\mathrm{p} \leq 0.05)$.

\section{Analysis of miRNA expression by qRT-PCR array}

During analysis of the miRNA expression in isolated salivary EXOs in all studied groups, two miRNAs (miR-200a and miR-134) were identified.

MiR-134 was found to up-regulated in OSCC isolated EXOs when compared to smoker and control groups. Highly significant increase was observed in miR-134 concentration in OSCC patients in a comparison to the smoker group and control one ( $\mathrm{p} \leq 0.00001)$, (Table 2, graph 2).

On the contrary, miR-200a was found to be down-regulated in OSCC isolated EXOs when compared to smoker and control groups. Highly significant decrease was observed in miR-200a concentration in OSCC patients in a comparison to the other studied groups $(\mathrm{p} \leq 0.00001)$, (Table 2, graph 2).

No statistical differences were found between the two identified miRNA profiles in OSCC patients and the other tested groups $(\mathrm{p} \leq 0.2)$.

Our study revealed that miR-134 expression was higher in high grade OSCC (5.979 \pm 0.916$)$ than low grade ones $(4.6341 \pm 0.583)$ while miR200a expression was higher in low grade tumors $(2.501 \pm 0.48)$ in contrast to high grade ones (1.773 \pm 0.247$)$. However, no statistical difference was revealed between different grades of OSCC. Strong positive correlation has been demonstrated between concentration of interleukins and expressed miRNAs in all studied groups $(\mathrm{R}=0.9045)$.

Moreover, no correlation was showed in our results between patients age and gender with the concentration of isolated salivary EXOs. 
TABLE (1) Mean values of IL- $1 \beta$ and IL- 8 concentrations measured in isolated salivary EXOs of all studied groups:

\begin{tabular}{|c|c|c|c|}
\hline Salivary Biomarker & Control Group & Smoker Group & OSCC Group \\
\hline \multirow{2}{*}{ IL-1 $\beta$} & $22.8 \pm 4.7539$ & $100.33 \pm 13.39652$ & $224.44 \pm 24.7038$ \\
\cline { 2 - 4 }$(\mathrm{pg} / \mathrm{ml})$ & \multicolumn{3}{|c|}{$\mathrm{f} \mathrm{ratio=}=235.92987$} \\
\cline { 2 - 4 } & $\mathrm{p}=0.00001^{* *}$ \\
\hline \multirow{2}{*}{ IL-8 } & $180.5 \pm 28.37428$ & $364 \pm 7.68615$ & $575.5556 \pm 116.783$ \\
\cline { 2 - 4 } & \multicolumn{3}{|c|}{$\mathrm{f} \mathrm{ratio}=45.05112$} \\
\cline { 2 - 4 } & $\mathrm{p}=0.00001^{* *}$ \\
\hline
\end{tabular}

**Highly significant

TABLE (2) Mean values of extracted miR-200a and miR-134 measured in isolated salivary EXOs of all studied groups:

\begin{tabular}{|c|c|c|c|}
\hline Salivary Biomarker & Control Group & Smoker Group & OSCC Group \\
\hline \multirow{3}{*}{ miR-200a } & $5.818056 \pm 0.830252$ & $3.785 \pm 0.456015$ & $2.110667 \pm 0.426652$ \\
\cline { 2 - 4 } & f ratio $=61.22368$ \\
\cline { 2 - 4 } & $2.030167 \pm 0.398726$ & $\mathrm{p}=0.00001 * *$ \\
\hline \multirow{3}{*}{ miR-134 } & $3.583833 \pm 0.376292$ & $5.294078 \pm 0.774052$ \\
\cline { 2 - 4 } & f ratio $=55.82431$ \\
\cline { 2 - 4 } & $\mathrm{p}=0.00001 * *$ \\
\hline
\end{tabular}

**Highly significant

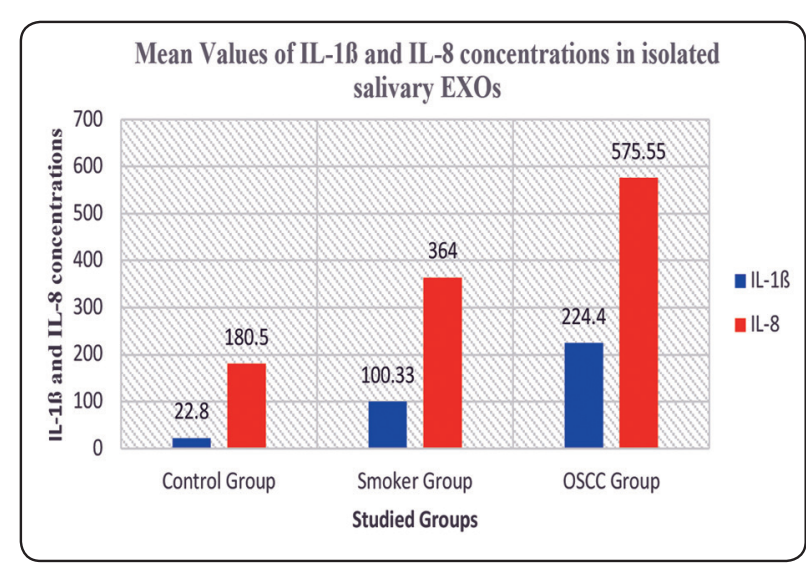

GRAPH (1) Mean Values of IL-1ß and IL-8 concentrations in isolated salivary EXOs of all studied groups.

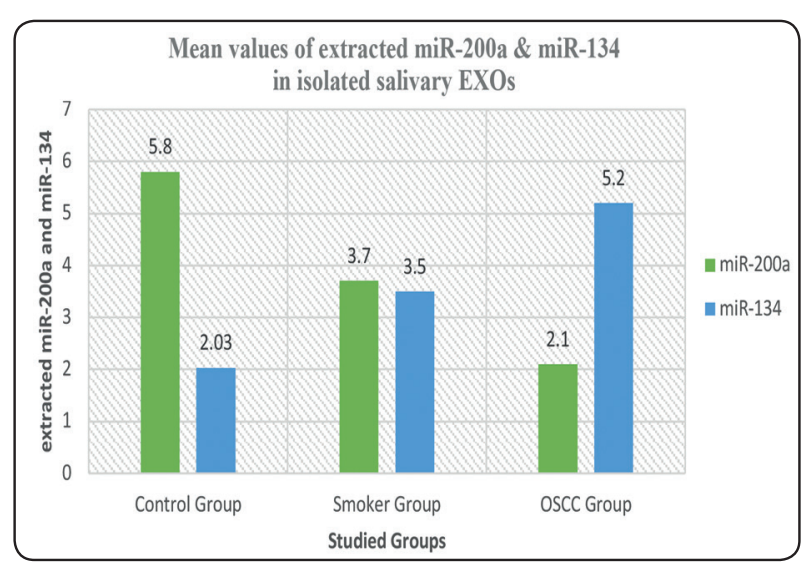

GRAPH (2) Mean values of extracted miR-200a and miR-134 in isolated salivary EXOs of all studied groups. 


\section{DISCUSSION}

Main concern of any cancer-screening program is to detect tumors at a stage early enough for successful treatment by using screening inexpensive tools with high sensitivity and specificity allowing sufficiently non-invasive widespread applicability ${ }^{(5)}$.

Saliva meets the demand for non-invasive, accessible and highly efficient diagnostic medium where the salivary transcriptome can be a suitable tool for the development of non-invasive diagnostic, prognostic and follow-up tests for cancer ${ }^{(2,21)}$.

Significant attention for extracellular vesicles especially EXOs secreted in saliva under a variety of pathophysiological and physiological conditions have gained as a conceivable source as cancer biomarkers ${ }^{(22)}$.

The tumor local environment is affected by tumor-derived EXOs carrying nucleic acids and biologically active proteins from their parent cells that in turn promoting cancer angiogenesis, invasion, progression and survival. Therefore, such derived EXOs can be used as molecular signatures of those cancer cells ${ }^{(13,23)}$.

The results obtained from the current work revealed that EXOs isolated from saliva of OSCC samples appeared as heterogeneous population with varying shapes, vesicle aggregation and numerous hollow large EXOs and to a less extent in smokers' samples. On the contrary to the homogeneous population without any apparent intervesicular fusion or aggregation was detected in healthy normal control samples. In addition, the size and concentration of salivary EXOs isolated from all participants demonstrated a slightly increase in OSCC patients compared to smoker patients and showed massive increase when compared to control healthy group.

These findings were in agreement with Gai et al. ${ }^{(12)}$ and Sharma et al. ${ }^{(24)}$ who reported that cancer EXOs populations displayed aggregated vesicles or clusters with variable morphology and sizes (ranging from 20-400 nm) compared to more circular ho- mogeneous morphology and a smaller size (ranging between 40 and $80 \mathrm{~nm}$ ) found in all normal salivary EXOs samples. Such differences were statistically significant in first study but no apparent differences in the second one.

Such increase in size and concentration of salivary EXOs in cancer patients compared to healthy controls in addition to morphological changes and higher intervesicular aggregation were hypothesized to be related to the differences in the binding affinity and adhesion between those derived from normal cells and cancer cells during the preparation of salivary samples for TEM analysis leading to either inherent overall increase in their size or their aggregation/fusion during or after excretion of the vesicles into the saliva ${ }^{(12,24)}$.

In the present study, a highly significant increase in salivary IL- $1 \beta$ concentration was observed in OSCC patients compared to smoker and healthy control groups. This finding was in accordance with the results of $\mathrm{Hu}$ et al. ${ }^{(25)}$ capillary reversed-phase liquid chromatography with quadruple time-offlight mass spectrometry, and Mascot sequence database searching. Immunoassays were used for validation of the candidate biomarkers on a new group of OSCC ( $n=48$ and Katakura ${ }^{(26)}$ who reported that significantly higher salivary IL- $1 \beta$ concentrations in patients with oral cancer compared to those with OPMDs and healthy controls.

Brailo et al. ${ }^{(8)}$ obtained similar results where salivary IL-1 $\beta$ recorded the highest concentration in OSCC patients compared to those with leukoplakia and control group which suggest the possibility for using such elevation in salivary biomarker for assessing the malignant transformation potential.

Salivary IL-8 was also significantly higher in OSCC patients of present study compared to smoker patients and control group which came in line with the study of Punyani and Sathawane ${ }^{(9)}$ and Rhodus et al. ${ }^{(27)}$ who found that the levels of salivary IL-8 in patients with OSCC were significantly higher than in patients with OPMDs and healthy controls. 
Similar results were revealed by Kaur and Jacobs ${ }^{(10)}$ where IL-8 level was higher in advanced stages of OMPDs than early stages. This was explained by excessive cell proliferation and activation of cellular actions instigated by chronic inflammation, which led to the induction of irreversible DNA damage ${ }^{(28)}$.

Our results also showed that the difference between analyzed salivary IL- $1 \beta$ and IL-8 concentrations in all tested groups was statistically significant. This was in consistent with the results obtained by Li et al. ${ }^{(7)}$ and St. John et al. ${ }^{(29)}$ who proven that salivary IL-8 to be the most significantly elevated transcript found in saliva of oral cancer patients compared to other cytokines tested especially IL-1 $\beta$.

The results of our study in addition to studies performed by Brailo et al. ${ }^{(8)}$, Rhodus et al. (27) and SahebJamee et al. ${ }^{(30)}$ indicated that increased concentrations of salivary IL- $1 \beta$ and IL- 8 among several cytokines represent the local production of oral cancer cells. Moreover, smoking is a proven risk factor of OPMDs. Both IL- $1 \beta$ and IL-8 are among the pro-inflammatory cytokines that released during the inflammatory response associated with smoking promote tumor growth ${ }^{(31,32)}$.

Moreover, tumor growth itself stimulates the inflammatory response, resulting in cyclic progression as cancer cells and tumor infiltrating lymphocytes can produce salivary IL- $1 \beta$ and IL-8. Upregulation of these cytokines stimulate other positive cell cycle regulators such as nuclear factor kappa B (NF$\varkappa \mathrm{B})$, signal transducer and activator of transcription (STAT) and mitogen-activated protein kinase/extracellular signal-regulated (ERK) pathway which in turn promotes cancer cell survival, proliferation and growth $^{(4,8,10,27)}$.

Based on the results of this study and previously mentioned roles of salivary cytokines, the increase in the production of salivary IL- $1 \beta$ and IL- 8 is considered to be marked feature of carcinogenesis that can be used as markers for early cancer detection and monitoring the malignant transformation of OPMDs.
MiRNAs are potentially very powerful and useful cancer biomarkers in salivary diagnostics especially for characterizing solid tumors than mRNA as they are differentially expressed in several cancer cells compared to normal cells and precisely discriminate poorly differentiated tumors better than mRNA profiling that produced highly inaccurate results on the same samples ${ }^{(4)}$.

Results of this study revealed highly significant increase in concentration of miR-134 in patients with OSCC isolated EXOs when compared to smoker and control groups. Peng et al. ${ }^{(14)}$, reported similar findings on testing miR-134 oncogenic effect during analysis of OPMD tissues and OSCC cell lines revealed an inverse correlation between miR134 expression and E-cadherin expression leading to increased cancer cell growth and migration. Moreover, lower miR-134 expression was recorded in higher grade tumors than lower grades but with no statistical differences and miR-134/E-cadherin expression was not associated with clinical stages of OSCC, tumor size or nodal metastasis, like our results.

Previous results were further supported by Liu et al. ${ }^{(15)}$ who reported an inverse association between miR-134 expression and WWOX gene in HNSCC tumor tissues. Highly expressed miR-134 result in inactivation of WWOX tumor suppressor gene which in turn contributes to carcinogenesis, cell invasion, nodal metastasis, mortality and poor survival.

In contrast to our results, Salazar et al. ${ }^{(33)}$ who develop a panel of saliva-based miRNA diagnostic biomarkers for the detection of HNSCC they observed down-regulation of miR-134. Such downregulation could be attributed to small sample size of tested groups or may be due to drug resistance of tested cases.

Regardless the controversy associated with the complicated roles of miR-134 in malignant transformation. Results of the current study and heterogeneous literature data up to date ascertain 
that the oncogenic role of miR-134 in OSCC cells responsible for malignant transformation, tumor initiation, proliferation and growth and even invasion and metastasis in several types of cancer especially OSCC ${ }^{(14,15)}$.

In current research, isolated EXOs from OSCC samples showed a highly significant decrease in miR-200a concentration compared to the smoker group and control one. This down-regulation was consistent with the findings of Park et al. ${ }^{(34)}$ who strongly suggested miR-200a as biomarker that can discriminate oral cancer patients from control subjects.

These is further supported by the study performed by Arunkumar et al. ${ }^{(18)}$ who studied ZEB1 and ZEB2 expression to evaluate the miR-200 family down-regulation in OSCC and tobacco smokers. They reported significant downregulation in miR-200a levels in OSCC patients where poorly differentiated tumors showed lower levels than welldifferentiated ones. Also, their study revealed significant downregulation in miR-200a levels in high risk patients with tobacco smoking when compared to normal group.

The miR-200 family associated with OSCC patients was downregulated compared to the normal tissue could be related to upregulation of EMT inducers, mainly ZEB1 and ZEB2 which led to modulation of Ecadherin and vimentin expression with subsequent EMT in oral tumors ${ }^{(18,34,35)}$.

Furthermore, it was assumed that the strong association between the miR-200a deregulation and tobacco smoking may be related to tobacco carcinogens that induced the malignant transformation through epigenetic silencing of miR-200 family which in turn led to EMT and stem celllike properties ${ }^{(18,36)}$.

Another study conducted by Wilkund et al. ${ }^{(37)}$ revealed lower miR-200a levels when analyzed miRNA expression and changes in DNA methylation in OSCC patients. They suggested that epigenetic mechanisms such as altered DNA methylation patterns may play an important role in silencing tumor-suppressive miRNAs leading to progressive accumulation of multiple genetic abnormalities that contributes to oral carcinogenesis, tumor invasion and metastasis, poor overall survival and resistance to therapy ${ }^{(38)}$.

In contrast to our results, salivary miR-200a had been reported to be differentially expressed with higher level in various OSCC cell lines compared to lower levels in OSCC patients ${ }^{(39,40)}$. This discrepancy could be due to the presence of salivary miRNAs in cell-free state compared with the ones in living cells where they may undergo more rapid degradation and have a shorter half-life during cell death, similar to the degradation of regulatory mRNAs ${ }^{(34,41,42)}$.

Therefore, observations from this study and previously published data indicate that miR-200 family members especially miR-200a are considered as tumor suppressor genes critical for the preservation of the epithelial phenotype, inhibition of EMT, tumor invasion and metastasis and have been deregulated in metastatic cells in different types of cancer.

\section{CONCLUSION}

Salivary biomarkers represent a promising noninvasive approach for early detection of oral cancer and monitoring the activity of non-cancerous disease. However, some issues need to be fixed for making such approach as a reliable method in clinical application. Moreover, validation of those OSCC salivary biomarker is still a major issue due lack of standardization for collection and storage of saliva samples. Therefore, further investigations are recommended to overcome these issues.

\section{Disclosure statement}

All authors declare that no conflict of interest.

\section{Research Highlights}

- Recent modalities such as collected human saliva can guarantee a high success rate of oral squamous cell carcinoma treatment. 
- Isolated salivary exosomes provide a stable and non-invasive route for evaluation of different salivary biomarkers which be a useful tool in early detection of oral cancer.

- The expression of microRNAs and interleukins in exosomes can serve as potential salivary biomarkers in early detection of oral cancer.

- Concentrations of both salivary IL-1 $1 \beta$ \& IL-8 demonstrated a highly significant increase in cancer patients compared to smoker and control groups.

- Concentrations of both salivary microRNA200a and microRNA-134 were upregulated and revealed a highly significant increase in cancer patients in comparison to other groups.

\section{REFERENCES}

1. Mazumder S, Datta S, Ray JG, Chaudhuri K, Chatterjee R. Liquid biopsy : miRNA as a potential biomarker in oral cancer. 2019;58(December 2018):137-45.

2. Saxena S, Sankhla B. A Review of Salivary Biomarker : A Tool for Early Oral Cancer Diagnosis. 2017;

3. Shah FD, Begum R, Vajaria BN, Patel KR, Patel JB, Shukla SN, et al. A review on salivary genomics and proteomics biomarkers in oral cancer. Indian J Clin Biochem. 2011;26(4):326-34.

4. Bano S, David MP, Indira AP. Salivary Biomarkers for Oral Squamous Cell Carcinoma, : An Overview. 2015;1(1):39-45.

5. Rapado-gonzález Ó, Majem B, Muinelo-romay L, Álvarez-castro A, Gil-moreno A, López-lópez R, et al. Journal of Cancer Human salivary microRNAs in Cancer. 2018;9.

6. Cheng J, Nonaka T. Salivary Exosomes as Nanocarriers for Cancer Biomarker Delivery. 2019;1-18.

7. Li Y, John MARS, Zhou X, Kim Y, Sinha U, Jordan RCK, et al. Salivary Transcriptome Diagnostics for Oral Cancer Detection. 2004;10(310):8442-50.

8. Brailo V, Vucicevic-boras V, Lukac J, Biocina-lukenda D, Zilic-alajbeg I. Salivary and serum interleukin 1 beta, interleukin 6 and tumor necrosis factor alpha in patients with leukoplakia and oral cancer. 2012;17(1).

9. Punyani SR, Sathawane RS. Salivary level of interleukin-8 in oral precancer and oral squamous cell carcinoma. 2013;517-24.
10. Kaur J, Jacobs R. Proinflammatory cytokine levels in oral lichen planus , oral leukoplakia , and oral submucous fibrosis. $2015 ; 171-5$

11. He Y, Lin J, Kong D, Huang M, Xu C, Kim TK, et al. Current state of circulating microRNAs as cancer biomarkers. Clin Chem. 2015;61(9):1138-55.

12. Gai C, Camussi F, Broccoletti R, Gambino A, Cabras M, Molinaro L, et al. Salivary extracellular vesicle-associated miRNAs as potential biomarkers in oral squamous cell carcinoma. 2018;1-11.

13. Kinoshita T, Yip KW, Spence T, Liu F-F. MicroRNAs in extracellular vesicles: potential cancer biomarkers. J Hum Genet. 2017 Jan;62(1):67-74.

14. Peng S, Tu H, Yang C, Wu C, Liu C. miR-134 targets PDCD7 to reduce E-cadherin expression and enhance oral cancer progression. 2018;2904(155):2892-904.

15. Liu C-J, Shen WG, Peng S-Y, Cheng H-W, Kao S-Y, Lin S-C, et al. miR-134 induces oncogenicity and metastasis in head and neck carcinoma through targeting WWOX gene. Int J cancer. 2014 Feb;134(4):811-21.

16. Leivonen SK, Sahlberg KK, Mäkelä R, Due EU, Kallioniemi O, Børresen-Dale AL, et al. High-throughput screens identify microRNAs essential for HER2 positive breast cancer cell growth. Mol Oncol. 2014;8(1):93-104.

17. Tamagawa S, Beder LB, Hotomi M, Gunduz M, Yata K, Grenman R, et al. Role of miR-200c/miR-141 in the regulation of epithelial-mesenchymal transition and migration in head and neck squamous cell carcinoma. Int J Mol Med. 2014 Apr;33(4):879-86.

18. Arunkumar G, Kuha A, Magendhra D, Manikandan M, Prasanna H, Rao S, et al. Dysregulation of miR - 200 family microRNAs and epithelial - mesenchymal transition markers in oral squamous cell carcinoma. 2018;649-57.

19. Menck K, Bleckmann A, Schulz M, Ries L, Binder C. Isolation and characterization of microvesicles from peripheral blood. J Vis Exp. 2017;2017(119):1-7.

20. van der Pol E, Coumans FAW, Grootemaat AE, Gardiner C, Sargent IL, Harrison P, et al. Particle size distribution of exosomes and microvesicles determined by transmission electron microscopy, flow cytometry, nanoparticle tracking analysis, and resistive pulse sensing. J Thromb Haemost. 2014;12(7):1182-92.

21. Kaczor-Urbanowicz KE, Martin Carreras-Presas C, Aro K, Tu M, Garcia-Godoy F, Wong DTW. Saliva diag- 
nostics - Current views and directions. Exp Biol Med. 2017;242(5):459-72

22. Raposo G, Stoorvogel W. Extracellular vesicles: Exosomes, microvesicles, and friends. J Cell Biol. 2013;200(4): $373-83$.

23. Javeed N, Mukhopadhyay D. Exosomes and their role in the micro-/macro-environment: A comprehensive review. J Biomed Res. 2017;31(5):386-94.

24. Sharma S, Gillespie BM, Palanisamy V, Gimzewski JK. Quantitative Nanostructural and Single-Molecule Force Spectroscopy Biomolecular Analysis of Human-SalivaDerived Exosomes. 2011;14394-400.

25. Hu S, Arellano M, Boontheung P, Wang J, Zhou H, Jiang $\mathrm{J}$, et al. Salivary proteomics for oral cancer biomarker discovery. Clin Cancer Res. 2008 Oct;14(19):6246-52.

26. Katakura A, Kamiyama I, Takano N, Shibahara T, Muramatsu $\mathrm{T}$, Ishihara $\mathrm{K}$, et al. Comparison of salivary cytokine levels in oral cancer patients and healthy subjects. Bull Tokyo Dent Coll. 2007 Nov;48(4):199-203.

27. Rhodus NL, Ho V, Miller CS, Myers S, Ondrey F. NFkappaB dependent cytokine levels in saliva of patients with oral preneoplastic lesions and oral squamous cell carcinoma. Cancer Detect Prev. 2005;29(1):42-5.

28. Rai B, Kaur J, Jacobs R, Singh J. Possible action mechanism for curcumin in pre-cancerous lesions based on serum and salivary markers of oxidative stress. 2010;52(2): 251-6.

29. St John MAR, Li Y, Zhou X, Denny P, Ho C-M, Montemagno $\mathrm{C}$, et al. Interleukin 6 and interleukin 8 as potential biomarkers for oral cavity and oropharyngeal squamous cell carcinoma. Arch Otolaryngol Head Neck Surg. 2004 Aug;130(8):929-35.

30. Sahebjamee M, Eslami M, Atarbashimoghadam F, Sarafnejad A. Salivary concentration of TNF $\alpha$, IL1 $\alpha$, IL6, and IL8 in oral squamous cell carcinoma. 2008;13(5):292-5.

31. Vesty A, Gear K, Biswas K, Radcliff FJ, Taylor MW, Douglas RG. Microbial and inflammatory - based salivary biomarkers of head and neck squamous cell carcinoma. 2018;(August):255-62.
32. Rathnayake N, Rathnayake N, Klinge B, Jansson H. Salivary biomarkers of oral health - a cross-sectional study. 2013;8:140-7.

33. Salazar CA, Maccallum P, Centre C, Nagadia R, Pandit P, Cooper-white J. Metadata of the article that will be visualized in OnlineFirst. 2014;(August).

34. Park NJ, Zhou H, Elashoff D, Henson BS, Kastratovic DA, Abemayor E, et al. Salivary microRNA : Discovery, Characterization , and Clinical Utility for Oral Cancer Detection. 2009;15(17):5473-7.

35. Shrivastava S, Steele R, Sowadski M, Crawford SE, Varvares M, Ray RB. head and neck cancer stem-like cells. $2015 ; 1-8$.

36. Tellez CS, Juri DE, Do K, Bernauer AM, Thomas CL, Damiani LA, et al. EMT and Stem Cell - Like Properties Associated with miR-205 and miR-200 Epigenetic Silencing Are Early Manifestations during CarcinogenInduced Transformation of Human Lung Epithelial Cells. 2011;3087-98.

37. Wiklund ED, Gao S, Hulf T, Sibbritt T, Nair S, Costea DE, et al. MicroRNA Alterations and Associated Aberrant DNA Methylation Patterns across Multiple Sample Types in Oral Squamous Cell Carcinoma. 2011;6(11).

38. Koutsaki M, Spandidos DA, Zaravinos A. Epithelial-mesenchymal transition-associated miRNAs in ovarian carcinoma, with highlight on the miR-200 family: prognostic value and prospective role in ovarian cancer therapeutics. Cancer Lett. 2014 Sep;351(2):173-81.

39. Jiang J, Lee EJ, Gusev Y, Schmittgen TD. Real-time expression profiling of microRNA precursors in human cancer cell lines. 2005;33(17):5394-403.

40. Tran N, McLean T, Zhang X, Zhao CJ, Thomson JM, O'Brien C, et al. MicroRNA expression profiles in head and neck cancer cell lines. Biochem Biophys Res Commun. 2007 Jun;358(1):12-7.

41. Khabar KSA. The AU-rich transcriptome: more than interferons and cytokines, and its role in disease. J Interferon Cytokine Res. 2005 Jan;25(1):1-10.

42. Yang H, Kong W, He L, Zhao J, Donnell JDO, Wang J, et al. MicroRNA Expression Profiling in Human Ovarian Cancer : miR-214 Induces Cell Survival and Cisplatin Resistance by Targeting PTEN. 2008;(2). 